\title{
Correction to: Prospective trial examining safety and efficacy of microcurrent stimulation for the treatment of sinus pain and congestion
}

\author{
Alan B. Goldsobel ${ }^{1,2,3}$, Niveditha Prabhakar ${ }^{2}$ and Blake T. Gurfein ${ }^{3,4^{*}}$
}

\author{
Correction to: Bioelectronic Med (2019) 5:18 \\ https://doi.org/10.1186/s42234-019-0035-x
}

The original version of this article (Goldsobel et al. 2019), published on 20 November 2019, contained incorrect data. In this Correction the affected parts of the article are shown.

Figure 4 contained incorrect data. The correct number is marked bold and shown below:

Daily microcurrent treatment reduces sinonasal congestion over 4 weeks. Sinonasal congestion was assessed weekly using the Congestion Quanitfier 7 (CQ7) instrument. Subjects that enrolled with moderate or worse congestion (CQ7 > 15, $N=\mathbf{2 4}$ ) exhibited significant reductions in congestion symptoms, compared to pretreatment, at all time points measured (a). Mean difference in CQ7 score from before treatment peaked at -8.8 $(-45.0 \%)$ points at week four (b). Data represented as mean \pm SEM. ${ }^{* * * * * * *} p<0.0001$, paired, two-sided t-test

Table 2 contained incorrect data. The correct data is shown below:

For $N=24$ subjects with $\mathrm{CQ} 7>15$ below are the corrected results:

Mean CQ7 Score: Enrollment, 19.8; Week 1, 15.4; Week 2, 13.7; Week 3, 12.4; Week 4, 11.0

Difference in CQ7 from Enrollment: Week 1, -4.4 (C195 -2.6 to -6.2); Week 2, -6.3 (C195 -4.2 to -8.4 ); Week 3, -7.4 (CI95 -4.8 to -10.1 ); Week $4,-8.8$ (CI95 -6.5 to -11.1$)$
Percentage Decrease in CQ7 from Enrollment: Week 1, -23.0\%; Week 2, -32.8\%; Week 3,-38.1\%; Week 4, $-45.0 \%$

Statistics remain unchanged at $P<0.0001$ for all data points.

\footnotetext{
Author details

${ }^{1}$ Allergy and Asthma Associates of Santa Clara Valley Research Center, San Jose, CA, USA. ${ }^{2}$ Stanford University School of Medicine, Stanford, CA, USA. ${ }^{3}$ University of California San Francisco, San Francisco, CA, USA. ${ }^{4}$ Tivic Health Systems, Inc., 750 Menlo Ave \#200, Menlo Park, CA 94025, USA.
}

Published online: 30 January 2020

\section{Reference}

Goldsobel AB, et al. Prospective trial examining safety and efficacy of microcurrent stimulation for the treatment of sinus pain and congestion. Bioelectronic Med. 2019;5:18. https://doi.org/10.1186/s42234-019-0035-x. 